\title{
Research Article \\ Finding the Roots of System of Nonlinear Equations by a Novel Filled Function Method
}

\author{
Wei-Xiang Wang, ${ }^{1}$ You-Lin Shang, ${ }^{2}$ Wei-Gang Sun, ${ }^{3}$ \\ and Ying Zhang ${ }^{4}$ \\ ${ }^{1}$ School of Science, Shanghai Second Polytechnic University, Shanghai 201209, China \\ ${ }^{2}$ School of Science, Henan University of Science and Technology, Luoyang 471003, China \\ ${ }^{3}$ School of Science, Hangzhou Dianzi University, Hangzhou 310018, China \\ ${ }^{4}$ School of Science, Zhejiang Normal University, Jinhua 321004, China
}

Correspondence should be addressed to Wei-Xiang Wang, zhesx@126.com

Received 20 April 2011; Revised 2 October 2011; Accepted 2 October 2011

Academic Editor: Alexander I. Domoshnitsky

Copyright (c) 2011 Wei-Xiang Wang et al. This is an open access article distributed under the Creative Commons Attribution License, which permits unrestricted use, distribution, and reproduction in any medium, provided the original work is properly cited.

We present a novel filled function approach to solve box-constrained system of nonlinear equations. The system is first transformed into an equivalent nonsmooth global minimization problem, and then a new filled function method is proposed to solve this global optimization problem. Numerical experiments on several test problems are conducted and the computational results are also reported.

\section{Introduction}

Consider the system of nonlinear equations of the form: (SNE) : $F(x)=0, x \in X$, where the mapping $F: R^{n} \rightarrow R^{m}$ is continuous, and $X \subset R^{n}$ is a box set.

Denote $F(x)=\left(f_{1}(x), f_{2}(x), \ldots, f_{m}(x)\right)^{T}$, and let $f(x)=\sum_{k=1}^{m}\left|f_{k}(x)\right|$. To obtain the roots of (SNE), we may solve the following global optimization problem: $(P): \min _{x \in X} f(x)$. Obviously, if (SNE), has at least one root, then the global optimizers of the problem $(P)$ with the zero function value correspond to the roots of (SNE).

There are many theories and algorithms devoted to global optimization. Among of them, filled function method is a particularly useful tool (see [1-7]). The filled function method is originally introduced to solve smooth global optimization problem. In this paper, it is extended to solve nonsmooth global optimization problem $(P)$. The filled function method for nonsmooth global optimization contains two phases: local minimization and filling. The local minimization phase is intended to search for a local optimizer $x^{*}$ of the problem $(P)$, and the filling phase aims to identify an improved initial point by minimizing the following 
filled function constructed at $x^{*}:(\mathrm{FFP}) \min _{x \in X} F\left(x, x^{*}\right)$. The two phases are performed alternatively until no better optimizer can be found, and then the current optimizer is regarded as a global minimizer.

Comparing with the filled functions proposed in [1-7], our filled function has the following features.

(1) Since the functions $f_{i}(i=1, \ldots, m)$ in this paper are assumed to be Lipschitz continuous only, and not necessarily to be continuous differentiable, so the problem $(P)$ is a nonsmooth global optimization problem. Our filled function approach is proposed to tackle this kind of nonsmooth global optimization problem. Whereas these filled function methods proposed in the literature are mainly used for solving smooth global optimization problem.

(2) Although the filled function presented in [2] can be extended to solve nonsmooth global optimization problem, it has some disadvantages, such as it has two dependent parameters which are difficult to determine and hence make its algorithmic realization be fairly complicated. However, our proposed filled function contains only one parameter, which is easier to be appropriately chosen, and thus overcomes the disadvantages mentioned above in a certain extent.

(3) If each $f_{i}$ is continuous differentiable and $f(x)=\sum_{k=1}^{m} f_{k}^{2}(x)$, then problem $(P)$ is a smooth optimization problem. In this case, our proposed filled function method can also be used to solve this smooth problem, just as the above-mentioned filled function methods can do. Therefore, our filled function method is a natural extension of these filled function methods in the literature, and it has more scope of applications in the field of global optimization.

The paper is organized as follows: in Section 2, we make several assumptions on the problem. In Section 3, we present a novel filled function and investigate its properties. In Section 4, we describe the filled function algorithm. In Section 5, we make a numerical test. And, at last, in Section 6, we give our conclusion.

\section{Basic Knowledge}

In this section, we give a summary of main results on nonsmooth analysis, and define filled function for the problem $(P)$. For more details, we refer the reader to [8].

Definition 2.1. Let $f$ be Lipschitz with constant $L$ at the point $x$, the generalized gradient of $f$ at $x$ is defined as

$$
\partial f(x)=\left\{\xi \in R^{n}:\langle\xi, d\rangle \leq f^{0}(x ; d), \quad \forall d \in R^{n}\right\},
$$

where $f^{0}(x ; d)=\limsup _{y \rightarrow x, t \downarrow 0}(f(y+t d)-f(y)) / t$ is the generalized directional derivative of $f(x)$ in the direction $d$ at $x$.

Lemma 2.2. Let $f$ be Lipschitz with constant $L$ at the point $x$, then

(a) $f^{0}(x ; d)$ is finite, sublinear and satisfies $\left|f^{0}(x ; d)\right| \leq L\|d\|$,

(b) as a function of $(x, d), f^{0}(x ; d)$ is super-semicontinuous; as a function of $d$, it is Lipschitz with constant $L$, 
(c) $\partial \Sigma s_{i} f_{i}(x) \subseteq \Sigma s_{i} \partial f_{i}(x), \forall s_{i} \in R$,

(d) $\partial f(x)$ is a nonempty compact convex set, and to any $\xi \in \partial f(x)$, one has $\|\xi\| \leq L$,

(e) $\forall d \in X, f^{0}(x ; d)=\max \{\langle\xi, d\rangle: \xi \in \partial f(x)\}$.

Consider the problem $(P)$. To extend the filled function for smooth global optimization to nonsmooth case, throughout this paper, we make the following assumptions.

Assumption 1. The function $f_{i}(x)(i=1,2, \ldots, m)$ is Lipschitz continuous on $X$ with a rank $L_{i}>0$. Then $f(x)$ is Lipschitz continuous with a rank $L=\sum_{i=1}^{m} L_{i}$.

Assumption 2. The value of $f(x)$ for $x$ on the boundary of $X$ is greater than that of $f(x)$ for any $x$ within $X$.

Note that Assumption 2 implies that all optimizers of $(P)$ lie in the interior of $X$.

Assumption 3. The problem (SNE) has at least one root.

Definition 2.3. A function $P\left(x, x^{*}\right)$ is called a filled function of $f(x)$ at a local minimizer $x^{*}$, if it has the following properties:

(1) $f(x)$ is a strictly maximizer of $P\left(x, x^{*}\right)$,

(2) for any $x \in S_{1}=\left\{x \in X \backslash x^{*}: f(x) \geq f\left(x^{*}\right)\right\}$, one has $0 \notin \partial P\left(x, x^{*}\right)$,

(3) if $x^{*}$ is not a global minimizer of $(P)$, then $P\left(x, x^{*}\right)$ has at least one minimizer in the region $S_{2}=\left\{x \in X: f(x)<f\left(x^{*}\right)\right\}$.

\section{A New Filled Function and Its Properties}

Let $x^{*}$ be a local minimizer of $(P), L$ the Lipschitz rank of $f(x)$, and $D=\max _{x_{1}, x_{2} \in X}\left\|x_{1}-x_{2}\right\|$. Define

$$
F\left(x, x^{*}, r\right)=\left(\arctan f(x)-\frac{1}{5} \arctan f\left(x^{*}\right)\right) \exp \left(\frac{r}{\left\|x-x^{*}\right\|+1}\right),
$$

where $r>0$ is a parameter.

Next, we will prove that $F\left(x, x^{*}, r\right)$ is a filled function under mild conditions.

Lemma 3.1. Let $g(t)=\left(L t+(4 / 5) \arctan f\left(x^{*}\right)\right) \exp (r /(t+1))$, where $0 \leq t \leq D$. If $r$ is an appropriately large such that $r>\left(5 L(D+1)^{2} / 4 \arctan f\left(x^{*}\right)\right)$, then $g(t)$ is strictly monotone decreasing on $[0, D]$. 
Proof. Since $r>\left(5 L(D+1)^{2} / 4 \arctan f\left(x^{*}\right)\right)$, then we have

$$
\begin{aligned}
g^{\prime}(t) & =\exp \left(\frac{r}{t+1}\right)\left[L-\left(L t+\frac{4}{5} \arctan f\left(x^{*}\right)\right) \frac{r}{(t+1)^{2}}\right] \\
& \leq \exp \left(\frac{r}{t+1}\right)\left[L-\frac{4}{5} \arctan f\left(x^{*}\right) \frac{r}{(t+1)^{2}}\right] \\
& \leq \exp \left(\frac{r}{t+1}\right)\left[L-\frac{4}{5} \arctan f\left(x^{*}\right) \frac{r}{(D+1)^{2}}\right]<0
\end{aligned}
$$

which implies that $g^{\prime}(t)<0$, and $g(t)$ is strictly monotone decreasing on $[0, D]$.

Theorem 3.2. Let $x^{*}$ be a local minimizer of $f(x)$ with $f\left(x^{*}\right)>0$. Suppose that Assumption 2 holds and $r>0$ is appropriately large such that $r>\left(5 L(D+1)^{2} / 4 \arctan f\left(x^{*}\right)\right)$, then $x^{*}$ is a strict local maximizer of $F\left(x, x^{*}, r\right)$.

Proof. Since $x^{*}$ is a local minimizer of $f(x)$ over $X$, there exists a neighborhood $O\left(x^{*}, \delta\right)$ of $x^{*}$ with $\delta>0$ such that $f(x) \geq f\left(x^{*}\right)$ for all $x \in O\left(x^{*}, \delta\right) \cap X$.

For any $x \in O\left(x^{*}, \delta\right) \cap X$ with $x \neq x^{*}$, if $r>0$ is appropriately large such that $r>$ $\left(5 L(D+1)^{2} / 4 \arctan f\left(x^{*}\right)\right)$, then, by Lemma 3.1 and the fact that the following inequality holds for $b \geq a$,

$$
\arctan b-\arctan a \leq b-a,
$$

we have that

$$
\begin{aligned}
F\left(x, x^{*}, r\right) & =\exp \left(\frac{r}{\left\|x-x^{*}\right\|+1}\right)\left(\arctan f(x)-\frac{1}{5} \arctan f\left(x^{*}\right)\right) \\
& \leq \exp \left(\frac{r}{\left\|x-x^{*}\right\|+1}\right)\left(f(x)-f\left(x^{*}\right)+\frac{4}{5} \arctan f\left(x^{*}\right)\right) \\
& \leq \exp \left(\frac{r}{\left\|x-x^{*}\right\|+1}\right)\left(L\left\|x-x^{*}\right\|+\frac{4}{5} \arctan f\left(x^{*}\right)\right) \\
& <\exp (r) \frac{4}{5} \arctan f\left(x^{*}\right)=F\left(x, x^{*}, r\right) .
\end{aligned}
$$

Therefore, $x^{*}$ is a strict local maximizer of $F\left(x, x^{*}, r\right)$.

Theorem 3.3. Let $x^{*}$ be a local minimizer of $f(x)$ with $f\left(x^{*}\right)>0$. Suppose that $r>0$ is suitably large such that $r>\left(5 L(D+1)^{2} / 4 \arctan f\left(x^{*}\right)\right)$, then $0 \notin \partial F\left(x, x^{*}, r\right)$, for all $x \in S_{1}$. 
Proof. For any $x \in S_{1}$, that is, $f(x) \geq f\left(x^{*}\right)$ and $x \neq x^{*}$, we have

$$
\begin{aligned}
& \partial F\left(x, x^{*}, r\right) \\
& c \exp \left(\frac{r}{\left\|x-x^{*}\right\|+1}\right)\left[\frac{1}{1+f^{2}(x)} \partial f(x)\right. \\
& \left.-\frac{\arctan f(x)-(1 / 5) \arctan f\left(x^{*}\right)}{\left(\left\|x-x^{*}\right\|+1\right)^{2}} \frac{r\left(x-x^{*}\right)}{\left\|x-x^{*}\right\|}\right], \\
& \left\langle\partial F\left(x, x^{*}, r\right), \frac{x-x^{*}}{\left\|x-x^{*}\right\|}\right\rangle \\
& c \exp \left(\frac{r}{\left\|x-x^{*}\right\|+1}\right)\left[\left\langle\frac{1}{1+f^{2}(x)} \partial f(x), \frac{x-x^{*}}{\left\|x-x^{*}\right\|}\right\rangle\right. \\
& \left.-r \frac{\arctan f(x)-(1 / 5) \arctan f\left(x^{*}\right)}{\left(\left\|x-x^{*}\right\|+1\right)^{2}}\right] \\
& \leq \exp \left(\frac{r}{\left\|x-x^{*}\right\|+1}\right)\left[L-r \frac{(4 / 5) \arctan f\left(x^{*}\right)}{(D+1)^{2}}\right]<0 \text {, }
\end{aligned}
$$

since $\|\partial f(x)\| \leq L$, and $r>\left(5 L(D+1)^{2} / 4 \arctan f\left(x^{*}\right)\right)$.

It follows that $0 \notin \partial F\left(x, x^{*}, r\right)$ for all $x \in S_{1}$.

Theorem 3.4. Assume that Assumptions 2 and 3 hold, $x^{*}$ is a local optimizer with $f\left(x^{*}\right)>0$ and is not a global optimizer of $f(x)$ over $X$. Then there exists a point $x_{0} \in S_{2}$ such that $x_{0}$ is a minimizer of $F\left(x, x^{*}, r\right)$ over $X$.

Proof. Since Assumption 3 holds and $x^{*}$ is not a global minimizer of $f(x)$, there exists one point $x_{1}^{*}$ with $f\left(x_{1}^{*}\right)=0$ such that $f\left(x^{*}\right)>f\left(x_{1}^{*}\right)$. Denote the boundary of $X$ by $\partial X$. Then, by Assumption 2, for any $x \in \partial X$, it holds $F\left(x, x^{*}, r\right)>F\left(x_{1}^{*}, x^{*}, r\right)$. Suppose that $F\left(x, x^{*}, r\right)$ attains its minimum at the point $x_{0} \in X$, then we have

$$
\min _{x \in X} F\left(x, x^{*}, r\right)=\min _{x \in X \backslash \partial X} F\left(x, x^{*}, r\right)=F\left(x_{0}, x^{*}, r\right) \leq F\left(x_{1}^{*}, x^{*}, r\right)<0 .
$$

Since $X \backslash \partial X$ is an open set, we have $x_{0} \in S_{2}$. This completes the proof.

\section{Solution Algorithm}

Based on the discussion of the theoretical properties of the above-proposed filled function, we describe a filled function algorithm for solving (SNE) as follows.

Filled function algorithm:

\section{Initialization Step}

Let $r_{U}=10^{5}$ be a termination scalar. Choose an initial point $x_{1}$. Let $e_{1}, e_{2}, \ldots, e_{2 n}$ be the coordinate directions. Set $k=1$, and go to the main step. 
Main Step

(1) Start from $x_{1}$, solve the problem $(P)$ by using any nonsmooth local minimization procedure. Denote $x_{1}^{*}$ the obtained optimizer, and go to (2).

(2) Let $r=1$, construct the filled function $F\left(x, x^{*}, r\right)$ and go to (3).

(3) If $k \leq 2 n$, then set $x=x_{1}^{*}+0.1 e_{k}$, and use $x$ as an initial point for a nonsmooth local minimization method to find a local minimizer $x_{k}$ of the following problem: $\min _{x \in X} F\left(x, x^{*}, r\right)$. If $x$ attains the boundary of $X$ during minimization, then set $k=$ $k+1$, and repeat (3); otherwise, go to (4).

(4) If all the following conditions hold, then set $x=x_{k}, k=1$, use $x$ as an initial point to get another local solution $x_{2}^{*}$ of problem $(P)$, and go to (5); otherwise go to (6),

(a) $f\left(x_{k}\right)<f\left(x_{1}^{*}\right)$;

(b) $F\left(x_{k}, x_{1}^{*}, r\right)>F\left(x_{k-1}, x_{1}^{*}, r\right)$;

(c) $\left(x_{k}-x_{1}^{*}\right)^{T} \xi \geq 0, \forall \xi \in \partial F\left(x_{k}, x_{1}^{*}, r\right)$;

(d) $\|\xi\|<10^{-4}, \xi \in \partial F\left(x_{k}, x_{1}^{*}, r\right)$.

(5) If $f(x)<f\left(x_{1}^{*}\right)$, then set $x_{1}^{*}=x_{2}^{*}$, and go to (2); otherwise, go to (6).

(6) Increase $r$ by setting $r=10 r$. If $r \leq r_{U}$, then set $k=1$, and go to (2); otherwise, the algorithm is incapable of finding a better local minimizer, the algorithm stops, and $x_{1}^{*}$ is taken as a global minimizer.

Remarks. The above filled function algorithm consists of two phases: local minimization and filling. The local minimization phase is intended to minimize problem $(P)$ by applying nonsmooth local optimization algorithms, such as hybrid Hooke and Jeeves-Direct method for nonsmooth Optimization [9], mesh adaptive direct search algorithms for constrained optimization [10], bundle methods, and Powell's method. The filling phase aims to search for a point $x_{k}$ with $f\left(x_{k}\right)<f\left(x_{1}^{*}\right)$ by minimizing the filled function $F\left(x, x^{*}, r\right)$ over $X$. Once such kind of point is found in the process of minimization, the filling phase stops and the algorithm returns to the phase of local minimization. The two phases perform repeatedly until the certain conditions are satisfied.

\section{Numerical Experiment}

In this section, we perform the numerical experiments for four test problems by using the proposed filled function algorithm. All the numerical experiments are implemented in the FORTARN 95 environment. The hybrid Hooke and Jeeves-Direct method is used to search for the local minimizers of both the objective function and the filled function. Numerical results demonstrate that the proposed filled function approach is promising.

Problem 1. It holds that

$$
\begin{aligned}
& 4 x_{1}^{3}+4 x_{1} x_{2}+2 x_{2}^{2}-42 x_{1}-14=0 \\
& 4 x_{2}^{3}+4 x_{1} x_{2}+2 x_{1}^{2}-26 x_{2}-22=0 \\
& \text { s.t. }-5 \leq x_{1}, x_{2} \leq 5 .
\end{aligned}
$$


Table 1: Computational results for Problem 1.

\begin{tabular}{lcccc}
\hline$k$ & $x_{k}^{0}$ & $x_{k}^{*}$ & $f\left(x_{k}^{*}\right)$ & $F\left(x_{k}^{*}\right)$ \\
\hline 1 & $\left(\begin{array}{l}4.0000 \\
4.0000\end{array}\right)$ & $\left(\begin{array}{l}3.0000 \\
1.9999\end{array}\right)$ & $5.3020 \times 10^{-3}$ & $\left(\begin{array}{l}-2.0006 \times 10^{-3} \\
-3.3013 \times 10^{-3}\end{array}\right)$ \\
2 & $\left(\begin{array}{l}0.0866 \\
2.8843\end{array}\right)$ & $\left(\begin{array}{l}0.0866 \\
2.8842\end{array}\right)$ & $2.7777 \times 10^{-3}$ & $\left(\begin{array}{l}2.6285 \times 10^{-3} \\
1.4689 \times 10^{-4}\end{array}\right)$ \\
3 & $\left(\begin{array}{l}3.3852 \\
0.0738\end{array}\right)$ & $\left(\begin{array}{l}0.0866 \\
2.8843\end{array}\right)$ & $3.7337 \times 10^{-3}$ & $\left(\begin{array}{l}2.9451 \times 10^{-4} \\
2.4391 \times 10^{-3}\end{array}\right)$ \\
\hline
\end{tabular}

Table 2: Computational results for Problem 2.

\begin{tabular}{lcccc}
\hline$k$ & $x_{k}^{0}$ & $x_{k}^{*}$ & $f\left(x_{k}^{*}\right)$ & $F\left(x_{k}^{*}\right)$ \\
\hline 1 & $\left(\begin{array}{c}2.0000 \\
6.0000\end{array}\right)$ & $\left(\begin{array}{c}0.0000 \\
6.8753\end{array}\right)$ & $3.4105 \times 10^{-4}$ & $\left(\begin{array}{l}-1.0000 \\
-3.3252\end{array}\right)$ \\
2 & $\left(\begin{array}{c}9.9999 \times 10^{-6} \\
6.9667\end{array}\right)$ & $\left(\begin{array}{c}1.4354 \times 10^{-5} \\
6.9667\end{array}\right)$ & $7.1718 \times 10^{-5}$ & $\left(\begin{array}{c}1.1803 \times 10^{-7} \\
-7.1597 \times 10^{-5}\end{array}\right)$ \\
\hline
\end{tabular}

There are nine known roots as shown in [11]. Table 1 records the numerical results obtained by our algorithm for Problem 1.

Problem 2. It holds that

$$
\begin{aligned}
& 10^{4} x_{1} x_{2}-1=0 \\
& e^{-x_{1}}+e^{-x_{2}}-1.001=0 \\
& \text { s.t. } 5.49 \times 10^{-6} \leq x_{1} \leq 4.553,2.196 \times 10^{-3} \leq x_{2} \leq 18.21 .
\end{aligned}
$$

The algorithm successfully finds a root of the Problem 2: $\left(1.4564 \times 10^{-5}, 6.8753\right)$. Table 2 records its numerical results.

Problem 3. It holds that

$$
\begin{aligned}
& 1-2 x_{2}+0.2 \sin \left(4 \pi x_{2}\right)-x_{1}=0, \\
& x_{2}-0.5 \sin \left(2 \pi x_{1}\right)=0, \\
& \text { s.t. }-10 \leq x_{1}, x_{2} \leq 10 .
\end{aligned}
$$

The solution is $(1.8784,-0.3458)$. Table 3 records the numerical results of Problem 3. 
Table 3: Computational results for Problem 3.

\begin{tabular}{lcccc}
\hline$k$ & $x_{k}^{0}$ & $x_{k}^{*}$ & $f\left(x_{k}^{*}\right)$ & $F\left(x_{k}^{*}\right)$ \\
\hline 1 & $\left(\begin{array}{c}5.0000 \\
-3.0000\end{array}\right)$ & $\left(\begin{array}{c}4.7386 \\
-1.7417\end{array}\right)$ & 1.5191 & $\left(\begin{array}{l}-0.2762 \\
-1.2428\end{array}\right)$ \\
2 & $\left(\begin{array}{c}2.5975 \\
-0.3301\end{array}\right)$ & $\left(\begin{array}{c}2.5762 \\
-0.4315\end{array}\right)$ & 0.7627 & $\left(\begin{array}{l}-0.5615 \\
-0.2012\end{array}\right)$ \\
3 & $\left(\begin{array}{c}1.7394 \\
-0.4335\end{array}\right)$ & $\left(\begin{array}{c}1.8784 \\
-0.3458\end{array}\right)$ & $2.2341 \times 10^{-4}$ & $\left(\begin{array}{c}-1.1137 \times 10^{-4} \\
1.1203 \times 10^{-4}\end{array}\right)$ \\
\hline
\end{tabular}

Table 4: Computational results for Problem 4.

\begin{tabular}{ccccc}
\hline$k$ & $x_{k}^{0}$ & $x_{k}^{*}$ & $f\left(x_{k}^{*}\right)$ & $F\left(x_{k}^{*}\right)$ \\
\hline 1 & $\left(\begin{array}{c}-1.0000 \\
1.0000 \\
-1.00000 \\
1.0000 \\
-1.0000\end{array}\right)$ & $\left(\begin{array}{l}1.0054 \\
1.0055 \\
1.0053 \\
1.0055 \\
0.9739\end{array}\right)$ & $8.8951 \times 10^{-3}$ & $\left(\begin{array}{c}1.0008 \times 10^{-3} \\
1.1007 \times 10^{-3} \\
9.0007 \times 10^{-4} \\
1.1003 \times 10^{-3} \\
-4.7932 \times 10^{-3}\end{array}\right)$ \\
2 & $\left(\begin{array}{c}0.9935 \\
0.9933 \\
1.0016 \\
1.0133 \\
1.0127\end{array}\right)$ & $\left(\begin{array}{l}0.9990 \\
0.9989 \\
0.9991 \\
0.9996 \\
1.0042\end{array}\right)$ & $1.7952 \times 10^{-3}$ & $\left(\begin{array}{c}-2.0011 \times 10^{-4} \\
-3.0009 \times 10^{-4} \\
-1.0004 \times 10^{-4} \\
4.0007 \times 10^{-4} \\
7.9011 \times 10^{-4}\end{array}\right)$ \\
\hline
\end{tabular}

Problem 4. It holds that

$$
\begin{aligned}
& 2 x_{1}+x_{2}+x_{3}+x_{4}+x_{5}-6=0 \\
& x_{1}+2 x_{2}+x_{3}+x_{4}+x_{5}-6=0 \\
& x_{1}+x_{2}+2 x_{3}+x_{4}+x_{5}-6=0, \\
& x_{1}+x_{2}+x_{3}+2 x_{4}+x_{5}-6=0, \\
& x_{1} x_{2} x_{3} x_{4} x_{5}-1=0, \\
& \text { s.t. }\left|x_{i}\right| \leq 2, \quad i=1,2, \ldots, 5 .
\end{aligned}
$$

The known solutions of Problem 4 in [11] are $(1,1,1,1,1)$ and $(0.916,0.916,0.916,0.916$, 0.916). Table 4 records the numerical results of Problem 4 .

The symbols used in the tables are given below:

$k$ : the iteration number in finding the $k$ th local minimizer,

$x_{k}$ : the $k$ th initial point to find the $k$ th local minimizer,

$x_{k}^{*}$ : the $k$ th local minimizer,

$f\left(x_{k}^{*}\right)$ : the function value of $f(x)$ at the $k$ th local minimizer,

$F\left(x_{k}^{*}\right)$ : the function value of $F(x)$ at the $k$ th local minimizer. 


\section{Conclusions}

In this paper, we developed a global minimization method based on filled function to identify the roots of the system of nonlinear equations. By converting a system nonlinear equations into an equivalent nonsmooth global minimization problem, we manage to search for a root or an appropriate root of the system of nonlinear equations by solving the formulated global minimization problem. A new filled function algorithm is proposed to solve the formulated global minimization problem. Numerical experiments for several test problems verify that our proposed algorithm is promising.

\section{Acknowledgments}

The authors are very grateful to the anonymous referees and the editor for their extremely valuable comments and suggestions, which have contributed to significant improvements of this paper. This paper was partially supported by the NNSF of China under Grant nos. 10971053 and 11001248, and by the SEDF under Grant no. 12YZ178.

\section{References}

[1] R. P. Ge and Y. F. Qin, "A class of filled functions for finding global minimizers of a function of several variables," Journal of Optimization Theory and Applications, vol. 54, no. 2, pp. 241-252, 1987.

[2] R. P. Ge, "Theory of filled function method for finding global minimizers of nonlinearly constrained minimization problems," Journal of Computational Mathematics, vol. 5, no. 1, pp. 1-9, 1987.

[3] X. Liu, "Finding global minima with a computable filled function," Computers and Operations Research, vol. 31, pp. 61-80, 2004.

[4] L. S. Zhang, "On the solving global optimization approach from local to global," Journal of Chongqing Normal University, vol. 26, pp. 1-6, 2009.

[5] C. J. Wang, R. H. Luo, K. Wu, and B. Han, "A new filled function method for an unconstrained nonlinear equation," Journal of Computational and Applied Mathematics, vol. 235, no. 6, pp. 1689-1699, 2011.

[6] L.S. Zhang, C. K. Ng, D. Li, and W. W. Tian, "A new filled function method for global optimization," Journal of Global Optimization, vol. 28, no. 1, pp. 17-43, 2004.

[7] Y. J. Yang and Y. L. Shang, "A new filled function method for unconstrained global optimization," Applied Mathematics and Computation, vol. 173, no. 1, pp. 501-512, 2006.

[8] F. H. Clarke, Optimization and Nonsmooth Analysis, John Wiley \& Sons, New York, NY, USA, 1983.

[9] C. J. Price, B. L. Robertson, and M. Reale, "A hybrid Hooke and Jeeves-direct method for non-smooth optimization," Advanced Modeling and Optimization, vol. 11, no. 1, pp. 43-61, 2009.

[10] C. Audet and J. E. Dennis, Jr., "Mesh adaptive direct search algorithms for constrained optimization," SIAM Journal on Optimization, vol. 17, no. 1, pp. 188-217, 2006.

[11] C. A. Floudas, P. M. Pardalos, C. S. Adjiman, W. R. Esposito, Z. H. Gumus, and S. T. Harding, Handbook of Test Problems in Local and Global Optimization, Kluwer Academic, Dordrecht, The Netherlands, 1999. 


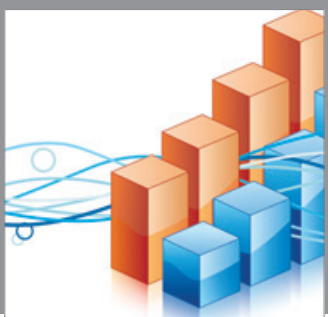

Advances in

Operations Research

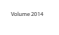

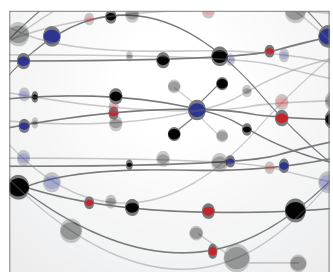

\section{The Scientific} World Journal
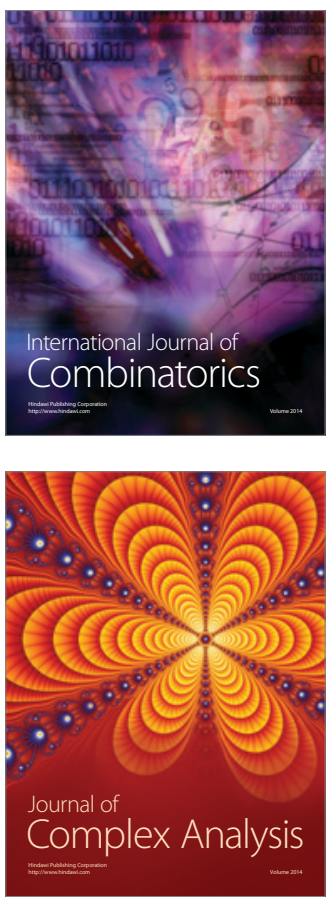

International Journal of

Mathematics and

Mathematical

Sciences
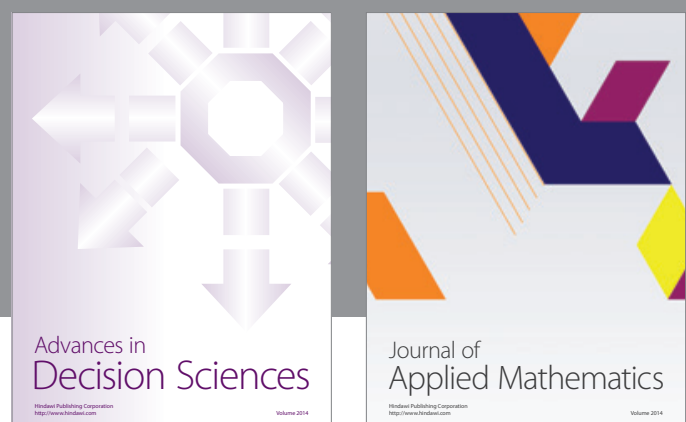

Journal of

Applied Mathematics
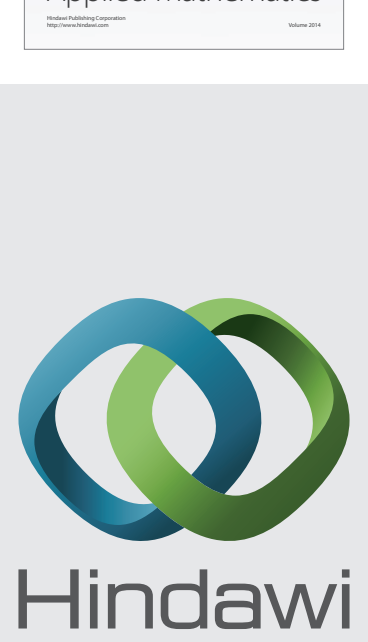

Submit your manuscripts at http://www.hindawi.com
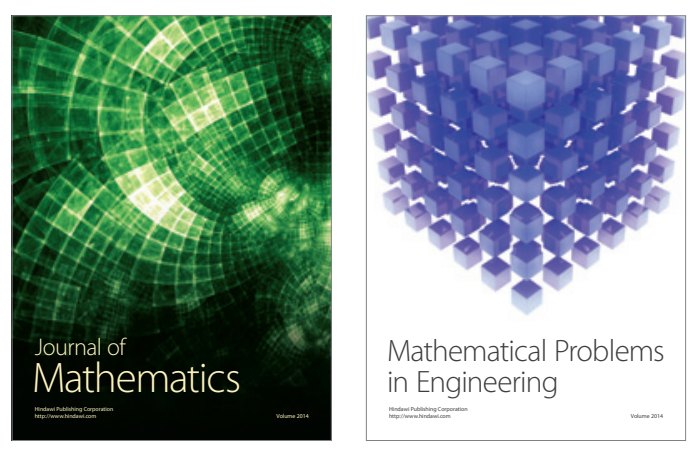

Mathematical Problems in Engineering
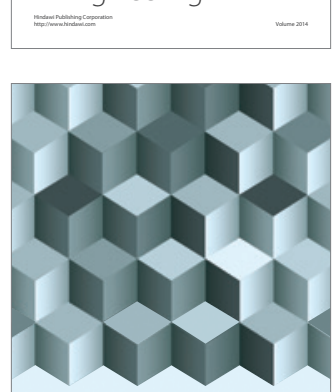

Journal of

Function Spaces
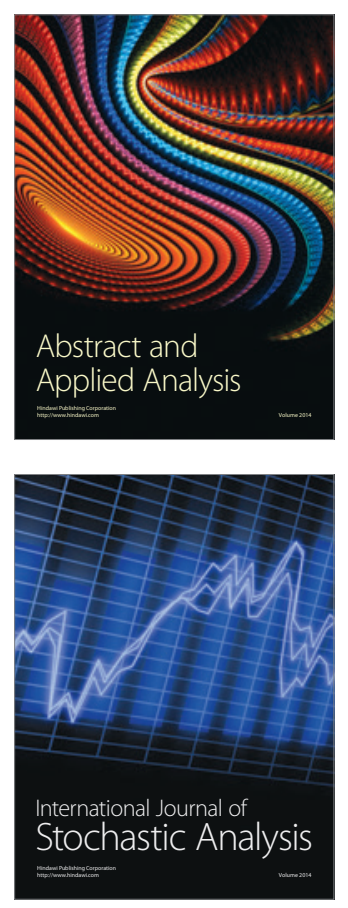

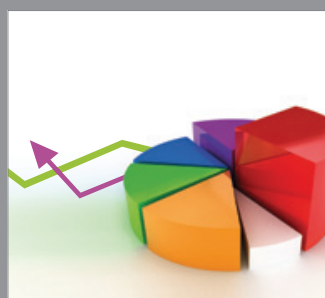

ournal of

Probability and Statistics

Promensencen
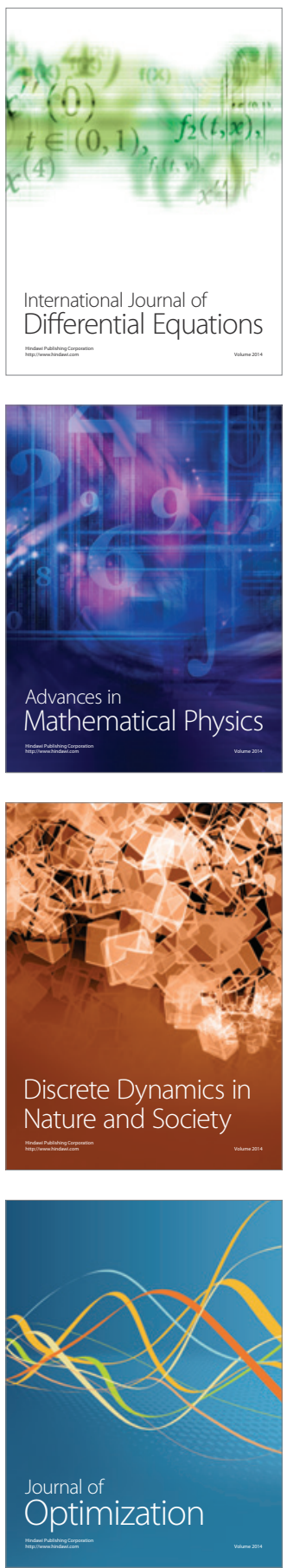